\title{
Inappropriate Discharges by Fourth Generation Implantable Cardioverter Defibrillators in Patients With Ventricular Arrhythmias
}

\author{
Takashi Washizuka, MD; Masaomi Chinushi, MD; Minoru Tagawa, MD; \\ Hidehiro Kasai, MD; Hiroshi Watanabe, MD; Yukio Hosaka, MD; \\ Fumio Yamashita, MD; Hiroshi Furushima, MD; Akira Abe, MD; \\ Hiroshi Watanabe, MD*; Junichi Hayashi, MD*; Yoshifusa Aizawa, MD
}

\begin{abstract}
The study prospectively investigated the incidence, cause and efficient management of inappropriate discharge by the fourth generation implantable cardioverter-defibrillator (ICD) system in 45 patients (mean age, $57 \pm 16$ years). During the follow-up period of $27 \pm 17$ months, 18 patients $(40 \%)$ experienced one or more inappropriate therapies: sinus and supraventricular tachycardia (15 patients) and $\mathrm{T}$ wave oversensing ( 3 patients). In the 15 patients, re-programming of the tachycardia detection interval and/or additional treatment with $\beta$-blocking agents were effective. In the 3 patients with $\mathrm{T}$ wave oversensing, the arrythmia was associated with an increase in $\mathrm{T}$ wave amplitude, change in $\mathrm{T}$ wave morphology and decreased $\mathrm{R}$ wave amplitude, and re-programming of the sensitivity of the local electrogram or changing the number of intervals to detect ventricular tachycardia decreased the number of inappropriate discharges in all 3 patients. In conclusion, inappropriate therapies are common problems in patients treated with the fourth generation ICD system, but most of them can be resolved using the dualchamber ICD system. However, in patients with T-wave oversensing, it is difficult to avoid inappropriate discharge completely, even if the dual-chamber ICD system is implanted. (Jpn Circ J 2001; 65: 927-930)
\end{abstract}

Key Words: Implantable cardioverter defibrillator; Inappropriate shock; T-wave oversensing

$\mathbf{T}$ he implantable cardioverter-defibrillator (ICD) effectively terminates ventricular tachycardia (VT) and ventricular fibrillation (VF) and can reduce the incidence of sudden cardiac death!, ${ }^{1,2}$ However, inappropriate discharges for rhythms other than VT or VF are the most frequent complication of this therapy. Tachycardia is identified by ICDs primarily from measurement of heart rate and/or the morphology of an intraventricular electrogram and so the ICD shock can be delivered for supraventricular tachycardia (SVT) or other causes $3^{3-6}$ Inappropriate therapies are not only uncomfortable and lead to early battery depletion, but can also cause fatal, malignant ventricular tachyarrhythmias? The ability of the fourth generation ICD devices to store exact electrograms (EGM) improves their diagnostic potential of inappropriate therapy and so we investigated the incidence and cause of inappropriate therapy by these ICDs to clarify which problems can be avoided by using the new dual-chamber ICDs.

\section{Methods}

Between April 1995 and May 2000, 45 patients (33 males, 12 females; mean age, $57 \pm 17$ years, range: $1-77$ )

(Received April 25, 2001; revised manuscript received July 11, 2001; accepted July 27, 2001)

First Department of Internal Medicine and *Department of Thoracic and Cardiovascular Surgery, Niigata University School of Medicine, Asahimachi, Niigata, Japan

Mailing address: Takashi Washizuka, MD, First Department of Internal Medicine, Niigata University School of Medicine, 1-754 Asahimachi, Niigata 951-8510, Japan. E-mail: washi@med.niigata-u. ac.jp were treated by implantation of a fourth generation ICD (Medtronic, MN, USA): 5 patients received the $7220 \mathrm{C}$ system, 6 had the $7221 \mathrm{Cx}$ system and 34 had the $7223 \mathrm{Cx}$ system. Right ventricular screw-in leads were used in 43 patients and tined leads were used in the other 2 patients. Both VT and VF were documented clinically in 7 patients, VF only in 11 patients and VT only in 23 patients; the remaining 4 patients had unexplained syncope. The left ventricular ejection fraction (LVEF) ranged from 19 to $70 \%$ $($ mean \pm SD, $43 \pm 15 \%)$. Sixteen patients had coronary artery disease, 7 had idiopathic dilated cardiomyopathy, 4 had hypertrophic cardiomyopathy, 3 had cardiac sarcoidosis, 3 had idiopathic left ventricular aneurysm, 2 had myotonic dystrophy, 6 patients had idiopathic VF, including 3 with Brugada syndrome, and there was 1 case each of long QT syndrome, arrhythmogenic right ventricular dysplasia, mitral valve insufficiency and congenital heart disease.

Before ICD implantation, an standard electrophysiological study (EPS) ${ }^{8}$ was performed in all patients in a nonsedated and post-absorptive state after written informed consent. All patients fulfilled one of the following criteria: (1) cardiac arrest caused by VF or VT, (2) spontaneous sustained VT when drug therapy or catheter ablation failed to prevent spontaneous or inducible ventricular tachyarrhythmias, or (3) unexplained syncope with clinically relevant VT or VF induced during the EPS when drug therapy or catheter ablation failed to prevent induction of ventricular tachyarrhythmias.

ICD implantation was performed under general anesthesia. An endocardial energy-delivering-pacing-sensing lead was placed at the right ventricular apex through the cephalic vein, and a biphasic defibrillation current was delivered 

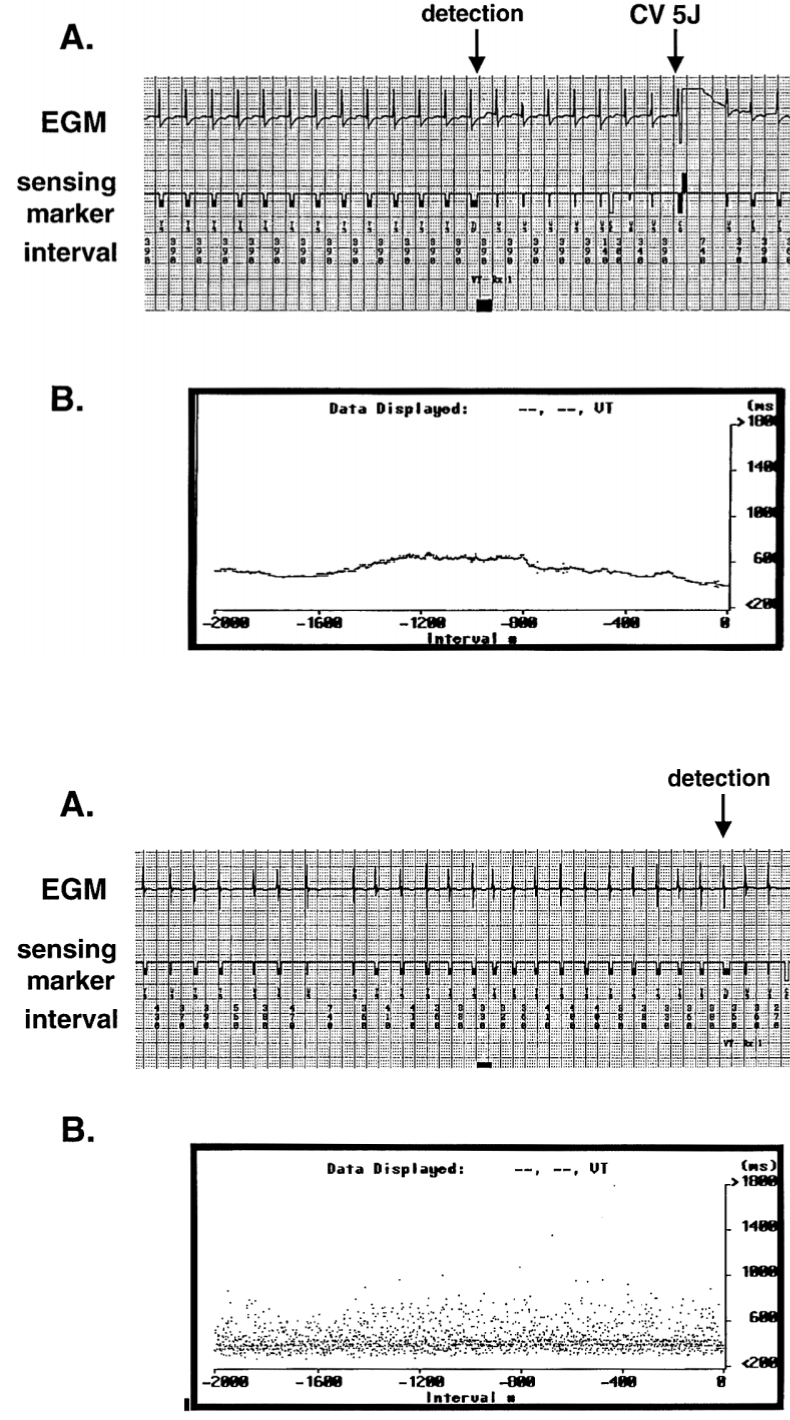

Fig 1. Inappropriate discharge caused by sinus tachycardia. (A) Intracardiac electrogram (EGM) shows regular tachycardia with a cycle length of $390 \mathrm{~ms}$ triggering a cardioversion (CV) of $5 \mathrm{~J}$. The morphology of this tachycardia was similar to that of sinus rhythm. (B) The RR interval trendgram before the discharge gradually shortened. between the lead and the ICD generator, which acted as the electrode. The following values were evaluated at implantation: (1) an R wave amplitude during spontaneous rhythm $(\geq 5 \mathrm{mV})$, (2) slew-rate $(\geq 0.75 \mathrm{~V} / \mathrm{s})$ and pacing threshold ( $\leq 1.0 \mathrm{~V}$ at $0.5 \mathrm{~ms}$ pulse width), as well as lead impedance

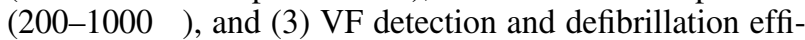
cacy. VF detection was tested at a programmed sensitivity of $1.2 \mathrm{mV}$ and confirmed that $75 \%$ of induced VF events were sensed by the ICD. P or T wave oversensing during sinus rhythm and pacing was tested at a sensitivity of 0.3 $\mathrm{mV}$. Defibrillation efficacy was twice confirmed as capable of terminating VF induced by $\mathrm{T}$-wave shock during the operation. Usually, the patients underwent a defibrillation test 1 or 2 weeks after the operation and a treadmill exercise test before hospital discharge to confirm that their heart rate during the exercise was lower than the interval of tachycardia detection.

All patients were seen routinely in our out-patient clinic at 3-month intervals, or as soon as possible after ICD discharges, for device interrogation and retrieval of stored EGMs. An ICD discharge was considered inappropriate when the stored EGMs showed a rhythm other than sustained VT or VF preceding the shock?
Fig 2. Inappropriate discharge caused by atrial fibrillation. (A) EGM shows irregular tachycardia with a mean cycle length of $380 \mathrm{~ms}$ triggering a cardioversion of $5 \mathrm{~J}$. The morphology of this tachycardia was similar to that after cardioversion. (B) The RR interval trendgram before discharge was variable. Abbreviations as in Fig 1.

\section{Results}

Incidence and Cause of Inappropriate Therapy by the ICD

In all patients, the ICD system was successfully implanted and only one patient developed frequent VT after the operation. Follow-up data were available for all patients. During a mean follow-up of $27 \pm 17$ months (range, $3-68), 32$ patients $(71 \%)$ received a total of 556 therapies from their devices: in 14 patients $(31 \%)$, therapies was appropriately delivered for sustained ventricular arrhythmias, 11 patients $(24 \%)$ received both appropriate and inappropriate therapies and in 7 patients $(16 \%)$, the device operated inappropriately at all times.

The causes of these inappropriate therapies included sinus tachycardia (ST) (9 patients), atrial tachycardia (AT) (2 patients), atrial fibrillation (AF) with rapid ventricular response (4 patients), T-wave oversensing ( 2 patients) and both ST and T wave oversensing (1 patient). The underlying heart diseases of these 18 patients were coronary artery disease ( 7 patients), dilated cardiomyopathy ( 2 patients), hypertrophic cardiomyopathy (2 patients), cardiac sarcoidosis (2 patients), idiopathic left ventricular aneurysm (2 patients) and others (3 patients). The mean LVEF of these patients was $41 \pm 12 \%$, which did not differ from the LVEF 


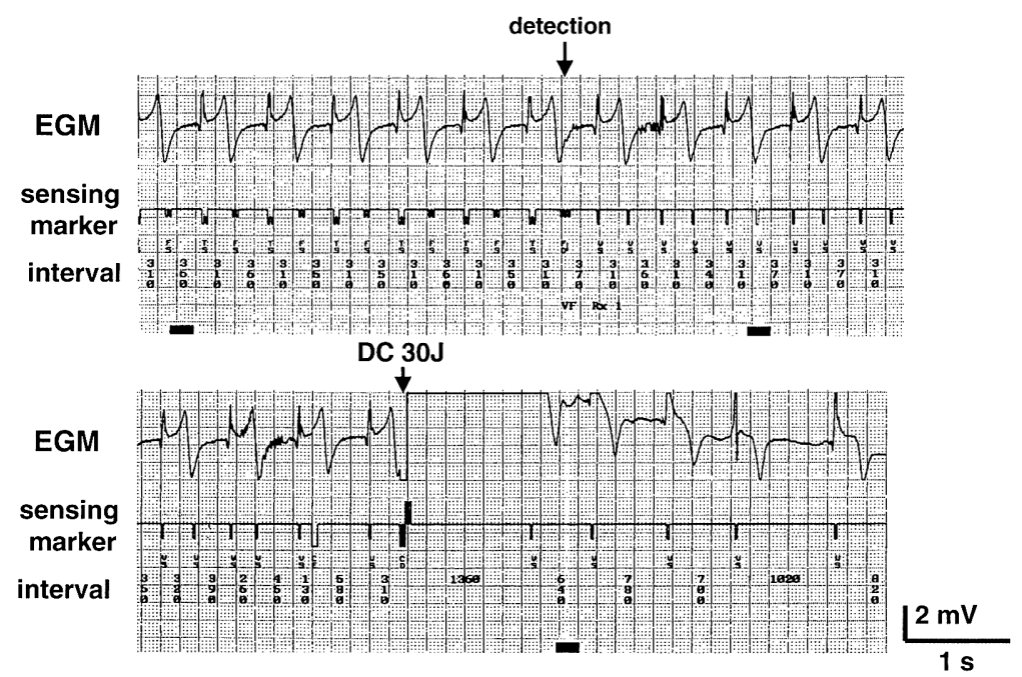

Fig 3. Inappropriate discharge caused by T-wave oversensing. EGM shows the double counting of Rwave and T-wave, and this oversensing triggered the ICD discharge. Sensed R-waves during T-wave oversensing decreased in size, while T-wave amplitude increased remarkably. DC, direct current shock; other abbreviations as in Fig 1.

of the patients with appropriate discharge. The time to the first inappropriate therapy after ICD implantation was $5 \pm 5$ months (range, 1-20). Fig 1 shows a case of inappropriate discharge for ST. In this patient, the rate of his clinical VT was 155 beats/min and the intraventricular EGM shows regular tachycardia with a cycle length of 390 ms triggering a cardioversion of $5 \mathrm{~J}$. The morphology of the stored EGM was similar to that of the sinus rhythm and, moreover, the RR interval gradually shortened before detection of the tachycardia (Fig 1B). Therefore, we diagnosed this tachycardia as ST. Fig 2 shows a case of inappropriate shock for $\mathrm{AF}$ with a rapid ventricular response. The EGM shows irregular ventricular activation with a mean cycle length of $380 \mathrm{~ms}$ triggering a cardioversion of $5 \mathrm{~J}$. The morphology of the EGM was similar to that after cardioversion, but as the RR interval before therapy was variable (Fig 2B), we diagnosed this as AF.

Fig 3 shows a case of T-wave oversensing. Both R- and $\mathrm{T}$-waves were counted as ventricular activation and satisfied the rate criteria of VF, and triggered a discharge of the ICD (30 J). The underlying heart diseases of the patients who had inappropriate discharge because of T-wave oversensing were hypertrophic cardiomyopathy, dilated cardiomyopathy and cardiac sarcoidosis. The cause of T-wave oversensing in the latter 2 patients was associated with marked diminution of R-wave amplitude and increased $\mathrm{T}$ wave amplitude within 2 months of implantation, despite the R-wave and T-wave amplitudes being satisfactory at implantation6 Moreover, there were no electrolyte abnormalites, and the position and loop of the lead had not changed on the chest X-rays.

\section{Management of Inappropriate Therapies by ICDs}

Of the 15 patients who received inappropriate ICD therapy for ST and SVT, including AT and AF with a rapid ventricular response, 12 were given medication to slow the sinus rate, to suppress atrioventricular conduction and/or to prevent recurrence of SVT (Fig 4): 11 were given $\beta$-blocking agents, 1 patient had disopyramide. The tachycardia detection interval or number of intervals to detect the ventricular tachyarrhythmia was reprogrammed in the other 3 patients and onset criteria was also used in one of these 3 . The 3 patients who received inappropriate discharges for T-wave oversensing underwent reprogramming of the sensitivity for the gain control, changing the number of

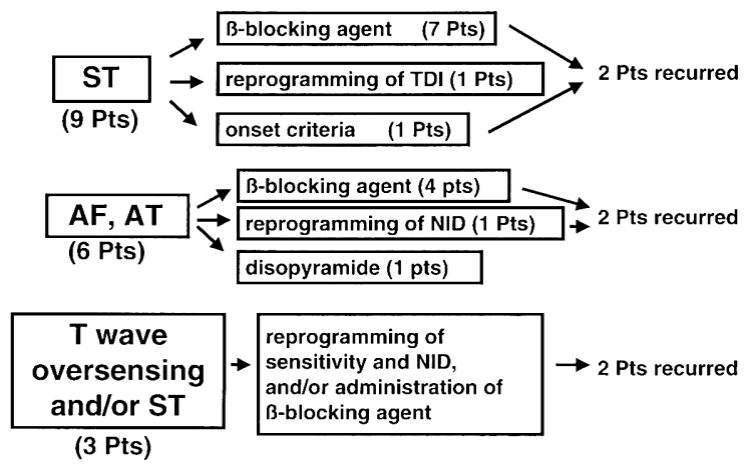

Fig 4. Management of inappropriate discharge. ST, sinus tachycardia; TDI, tachycardia detection interval; AF, atrial fibrillation; AT, atrial tachycardia; NID, number of intervals to detect ventricular tachyarrhyrhmia.

intervals to detect ventricular tachyarrhythmias, and/or slowing the heart rate with $\beta$-blocking agent to levels that did not satisfy the rate criteria for VF treatment, even if a T-wave was sensed. The repositioning or adding of the sensing lead was refused by these patients.

During a mean follow up of $12 \pm 8$ months after the first episode of inappropriate therapy, recurrence of inappropriate ICD therapy was observed in 6 of 18 patients $(33 \%)$ because of ST in 2 patients, T-wave oversensing in 2 patients, AT in 1 patient and AF with a rapid ventricular response in 1 patient. Medication to slow the heart rate and/or suppress atrioventricular conduction given to 4 patients who had recurrence of inappropriate ICD therapy for ST and SVT. The remaining 2 patients had reoperation recommended, but they refused.

\section{Discussion}

In the present study, inappropriate discharges from the fourth generation ICD were documented in $40 \%$ of patients and the major cause was unsuccessful discrimination of ST and SVT. Although most occurrences could be prevented by $\beta$-blocking agents or disopyramide and/or reprogramming the device, they recurred in 6 of 15 patients (40\%). The other cause of inappropriate discharge was $\mathrm{T}$-wave oversensing, which was observed in 3 patients and despite 


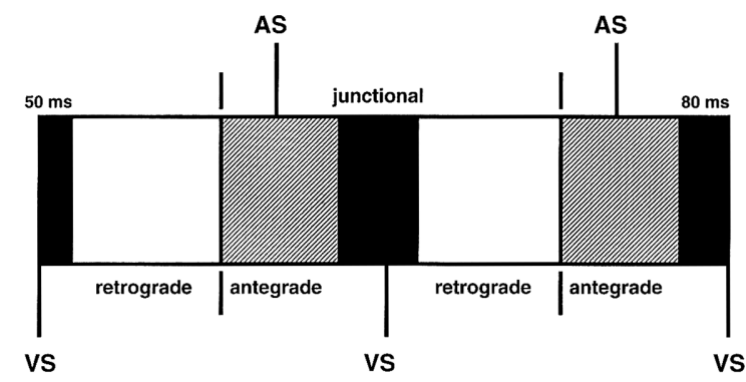

Fig 5. Atrioventricular (AV) pattern recognition for dual-chamber ventricular tachyarrhythmia detection algorithm. Each RR interval is divided into 4 zones to accommodate the expected atrial event during a tachyarrhythmia: antegrade $\mathrm{P}$ (sinus tachycardia), retrograde $\mathrm{P}$ (ventricular tachycardia), junctional P (in AV nodal tachycardia), and $\mathrm{AF}$ waves that can occur in various zones. The AV relationship is evaluated in a number of consecutive ventricular beats. AS, atrial sensed event; VS, ventricular sensed event.

additional medication and reprogramming of the ICD device, it recurred in 2 of them.

Previous studies of third generation ICD have reported a $20-40 \%$ incidence of inappropriate discharges ${ }^{3-5}$ The fourth generation ICDs have improved the storage capability of EGM during discharge and so the incidence of inappropriate discharges might be relatively high in our study. However, similar to the findings of other studies, the most common cause of inappropriate discharge in the present study was ST and SVT. The problem was that both inappropriate discharge and appropriate detection and termination of VT or VF occurred in the patients who had inappropriate discharge for ST and SVT. This is not surprising, because arrhythmia detection rates must be programmed in a zone with risks of inappropriate discharge for ST and SVT, particularly in patients with relatively slow VT. Actually, the majority of these patients had multiple VT morphologies $(3.0 \pm 1.3)$, and some of the VTs were slow and had overlapped with ST and SVT. In the present study, $40 \%$ of these patients had recurrent inappropriate discharge despite treatment. Some recently published studies have reported the advantages of using ICDs with a dual-chamber sensing capability!10,11 In those ICDs, arrhythmia classfication is chiefly based on the PR to RP relation, PP/RR regularity and evidence of ventriculoatrial (VA) conduction to discriminate ST/SVT and ventricular tachyarrhythmias. The PR to RP relation refers to the timing and the pattern of atrial events in relation to ventricular events. Each RR interval is divided into 4 zones (Fig 5) and using this classification, most of the inappropriate discharges for ST and SVT observed in the present study would have undoubtedly been prevented.

In the present study, the incidence of inappropriate discharge caused by T-wave oversensing was relatively high ( 3 of 45 patients). Although the exact mechanism was unclear, it may have been diseased myocardium that altered the R-wave amplitude, and the activity of the disease may have had an effect, because in another patient with cardiac sarcoidosis $^{6}$ incremental prednisolone administration de- creased the T-wave amplitude and the incidence of inappropriate discharges.

The theoretical solution to the problem of T-wave oversensing is repositioning or adding of a sensing lead, because the discharges synchronized to a sensed T-wave during sinus rhythm have the potential to induce severe ventricular tachyarrhythmias? Therefore, reoperation was strongly recommended for these patients, but they refused and so we subsequently performed reprogramming of the sensitivity for the gain control, the changing of the number of intervals to detect ventricular tachyarrhythmias and/or slowing the heart rate to levels that would not satisfy the rate criteria for VF treatment, even if a T-wave was sensed. However, these palliative measures could not suppress T-wave oversensing completely.

In conclusion, (1) inappropriate discharges are a frequent complication of ICD therapy, occurring in at least $40 \%$ of patients; (2) the cause of these shocks is most frequently SVT including sinus tachycardia; (3) the recurrence rate of inappropriate discharges caused by $\mathrm{T}$-wave oversensing is relatively high despite treatment; and (4) dual-chamber ICDs would undoubtfully decrease the frequency of inappropriate discharges, although T-wave oversensing is not prevented.

\section{References}

1. The AVID investigators: A comparison of antiarrhythmic drug therapy with implantable defibrillators in patients resuscitated from near-fatal ventricular arrhythmias. N Engl J Med 1997; 337: 15761583

2. Bocker D, Block M, Borggrefe M, Breithardt G: Defibrillators are superior to antiarrhythmic drugs in the treatment of ventricular tachyarrhythmias. Eur Heart J 1997; 18: 26-30

3. Grimm W, Florews BF, Marchlinski FE: Electrocardiographically documented unnecessary spontaneous shocks in 241 patients with implantable cardioverter-defibrillators. Pacing Clin Electrophysiol 1992; 15: $1667-1673$

4. Jafar MZ, Schloss EJ, Mehdirad AA, Keim S, Rist K, Siddiqui S, et al: Long-term survival and complications in patients with malignant ventricular tachyarrhythmias: Treatment with a nonthoracotomy implantable cardioverter defibrillator with or without a subcutaneous patch. Pacing Clin Electrophysiol 1997; 20: 1305-1311

5. Rosenqvist M, Beyer T, Block M, Dulk K, Minten J, Lindemans F: Adverse events with transvenous implantable cardioverter-defibrillators: A prospective multicenter study. Circulation 1998; 98: 663-670

6. Washizuka T, Chinushi M, Kasai H, Watanabe H, Tagawa M, Hosaka Y, et al: Inappropriate discharges of intravenous implantable cardioverter defibrillators due to T-wave oversensing. Jpn Circ J 2001; 65: 685-687

7. Plinski SL, Fahy GJ: The proarrhythmic potential of implantable cardioverter-defibrillators. Circulation 1995; 92: 1651-1664

8. Aizawa Y, Niwano S, Chinushi M, Tamura M, Kusano Y, Miyajima $\mathrm{T}$, et al: Incidence and mechanism of interruption of reentrant ventricular tachycardia with rapid ventricular pacing. Circulation 1992; 85: $589-595$

9. Hook BG, Marchlinski FE: Value of ventricular electrogram recordings in the diagnosis of arrhythmias precipitating electrical device shock therapy. J Am Coll Cardiol 1991; 17: 985-990

10. Wilkoff BL, Kuhlkamp V, Volosin K, Ellenbogen K, Waldecker B, Kacet S, et al: Critical analysis of dual-chamber implantable cardioverter-defibrillator arrhythmia detection: Results and technical considerations. Circulation 2001; 103: 381-386

11. Schoels W, Swerdlow CD, Jung W, Stein KM, Seidl K, Haffajee CJ, for the Worldwide JEWEL AF Investigators: Worldwide clinical experience with a new dual-chamber implantable cardioverter defibrillator system. J Cardiovasc Electrophysiol 2001; 12: 521-528 